\title{
Editorial: Bioceramics and/or Bioactive Glass-Based Composites
}

\author{
Valeria Cannillo ${ }^{1 *}$, Lech Pawlowski ${ }^{2}$, Sonia Fiorilli ${ }^{3}$ and Enrico Bernardo ${ }^{4}$ \\ ${ }^{1}$ Department of Engineering "Enzo Ferrari", University of Modena and Reggio Emilia, Modena, Italy, ${ }^{2}$ Faculté des Sciences et \\ Techniques, Université de Limoges, Limoges, France, ${ }^{3}$ Department of Applied Science and Technology, Politecnico di Torino, \\ Torino, Italy, ${ }^{4}$ Dipartimento di Ingegneria Industriale, University of Padua, Padua, Italy
}

Keywords: bioactive glass, bioceramic, composite, bioactivity, editorial

Editorial on the Research Topic

Bioceramics and/or Bioactive Glass-Based Composites

This Special Issue is dedicated to bioactive materials such as bioceramics, bioactive glasses and composites based on such constituents.

Bioactive materials are of fundamental importance: the demand for new materials suitable for healing of tissues and bone is dramatically increasing because of world population ageing.

Many attempts have been performed to design and produce materials with several concurrent features, such as adequate mechanical properties for the specific application and good biocompatibility and biological response to favor the regeneration of damaged tissues.

Among others, bioceramics and bioactive glass have proven to possess superior biological properties compared to other classes of materials. Hydroxyapatite and other calcium phophates have been widely used in the past years in both dentistry and orthopaedic surgery, mainly due to their good biocompatibility, osteoconduction and osseointegration. On the other hand, bioactive glasses have attracted a lot of interest, since they can bond with both hard and soft tissues and may have an antibacterial effect and stimulate new tissue formation.

Despite such interesting properties, bioceramics and bioactive glasses have some drawbacks, in particular their inherent brittleness that limits their usage as structural materials. Moreover, bioactive glasses are prone to crystallize during thermal treatments, which are often required to fabricate specific systems such as porous scaffolds or coatings; crystallization is reported to reduce or slow down the bioactivity of the final product.

Therefore, a smart approach is to produce composite materials, which can couple the favourable characteristics of bioceramics and bioactive glass with other materials that can for example satisfy the mechanical requirements or overcome problems related to fabrication. In fact, it is possible to tailor the properties of such composites by selectively varying the composition (i.e., the volume fractions of the two constituents) and thus produce devices with properties tailored for a specific clinical application. These hybrid composites (e.g., with a polymer phase) open new scenarios in tissue engineering and regenerative medicine. Composites based on bioactive glasses and bioceramics can be produced in various forms such as scaffolds, moldable implants, and surface coating, useful for many different applications.

This article collection focuses on the recent progress in new bioactive glass or bioceramic based composites. This article collection also highlights future challenges for the production of innovative materials for regenerative medicine.

We particularly welcomed contributions that included, but were not limited to, the following topics:

- Bioactive glass and/or bioceramic composites design 
- Bioactive coatings

- Innovative production routes

- New characterization techniques

- Novel applications of bioactive glass and/or bioceramic composites

- In vitro tests (cells) and in vivo tests

The papers dealt with the following issues:

Halaney et al. focused on "Chronic Brain Imaging Across a Transparent Nanocrystalline Yttria-Stabilized-Zirconia Cranial Implant." Repeated non-diffuse optical imaging of the brain is difficult. The authors demonstrated repeated brain imaging of five mice using both OCT and LSI across the WttB implant (transparent ceramic cranial implant called the Window to the Brain) over 4 weeks. The main objectives were to determine if the WttB implant allowed for chronic OCT imaging, and to shed further light on the question of whether optical access provided by the $\mathrm{WttB}$ implant remained stable over this duration in the body. The Window to the Brain implant allowed for stable repeated imaging of the mouse brain with Optical Coherence Tomography over 28 days, without loss of signal intensity.

Yang et al. focused on "Osteoporotic Goat Spine Implantation Study Using a Synthetic, Resorbable $\mathrm{Ca} / \mathrm{P} /$ S-Based Bone Substitute." The authors wanted to clarify whether the highly porous, resorbable $\mathrm{Ca} / \mathrm{P} / \mathrm{S}$-based bone substitute used in their study would still induce an osteoporotic bone when implanted into the osteoporotic vertebral defects of ovariectomized (OVX) goats. The histological examination revealed a newly formed trabecular bone network within the surgically-created defect of the implanted (OVX_IP) goat. The quick and nicely recovered trabecular architecture parameters observed in the OVX_IP goat indicated that the $\mathrm{Ca} / \mathrm{P} / \mathrm{S}$-based bone substitute material had a high potential to treat osteoporotic fractures.

$\mathrm{Hu}$ et al. focused on "Friction and Wear Behaviors of Reduced Graphene Oxide- and Carbon Nanotube-Reinforced Hydroxyapatite Bioceramics." Friction and wear properties play an important role in the long-term in vivo performance of loadbearing bioceramic implants. In this study, the friction and wear behavior of hydroxyapatite (HA) reinforced with reduced graphene oxide (rGO) and rGO + carbon nanotube (CNT) hybrids were studied by ball-on-disk tests to understand the effects of nanocarbon content and morphology on the composites' tribological behavior. The authors found that the incorporation of $\mathrm{rGO}$ and $\mathrm{rGO}+\mathrm{CNT}$ hybrids in $\mathrm{HA}$ bioceramic both improved both the friction and the wear behavior.

Song et al. focused on "In vivo Biocompatibility and Bioactivity of Calcium Silicate-Based Bioceramics in Endodontics." Due to their excellent biological features, a substantial number of calcium silicate-based bioceramics have been introduced into endodontics (i.e., a therapy that aims to preserve or repair the activity and function of pulp and periapical tissues); they increased the success rate of endodontic treatment. The authors described the in vivo biocompatibility and bioactivity of four types of calcium silicate-based bioceramics in endodontics.

Bernardo et al. focused on "Biosilicate ${ }^{(0)}$ Glass-Ceramic Foams From Refined Alkali Activation and Gel Casting." Biosilicate $^{\circledast}$ glass-ceramics are among the most valid alternatives to $45 \mathrm{~S} 5$ Bioglass. They combine a similar bioactivity and bioresorbability as the 45S5 with superior mechanical strength, due to the crystallization of a $\mathrm{Na}-\mathrm{Ca}$ silicate phase. Unlike in many crystallized bioglasses, the bioactivity is not degraded. The crystallization may be experienced upon viscous flow sintering of fine glass powders, thus configuring a sinter-crystallization process. The sintering/ crystallization combination was exploited for shaping highly porous bodies, to be used as scaffolds for bone tissue engineering, in the form of foams. The authors explored a gelcasting process, followed by sintering at $1,000^{\circ} \mathrm{C}$.

Finally, Melo et al. focused on "Processing of Sr2+ Containing Poly L-Lactic Acid-Based Hybrid Composites for Additive Manufacturing of Bone Scaffolds." The authors aimed at the development and process optimisation of two hybrid composite filaments, to be used as feedstock for the fused filament fabrication $3 \mathrm{D}$ printing process. Such hybrid composites indicated a shear thinning behaviour, ideal for the processing with fused filament fabrication, proving the potential of these materials to be processed into $3 \mathrm{D}$ structures for bone regeneration.

\section{AUTHOR CONTRIBUTIONS}

VC wrote the first draft of the manuscript. All authors contributed to manuscript revision, read, and approved the submitted version.

Conflict of Interest: The authors declare that the research was conducted in the absence of any commercial or financial relationships that could be construed as a potential conflict of interest.

Publisher's Note: All claims expressed in this article are solely those of the authors and do not necessarily represent those of their affiliated organizations, or those of the publisher, the editors and the reviewers. Any product that may be evaluated in this article, or claim that may be made by its manufacturer, is not guaranteed or endorsed by the publisher.

Copyright (C) 2021 Cannillo, Pawlowski, Fiorilli and Bernardo. This is an open-access article distributed under the terms of the Creative Commons Attribution License (CC $B Y)$. The use, distribution or reproduction in other forums is permitted, provided the original author(s) and the copyright owner(s) are credited and that the original publication in this journal is cited, in accordance with accepted academic practice. No use, distribution or reproduction is permitted which does not comply with these terms. 